\title{
Adopción del modelo educativo por competencias, desde la categoría de Campus de Bourdieu
}

Adoption of the competence-based educative model, from the Campus category of Bourdieu

\author{
Volumen 17, Número 2 \\ Mayo-Agosto \\ pp. 1-22
}

Este número se publica el $1^{\circ}$ de mayo de 2017

DOI: http://dx.doi.org/10.15517/aie.v17i1.28100

\author{
Elveri Figueroa Escudero \\ Verónica García Martínez
}

Revista indizada en REDALYC, $\underline{\text { SCIELO }}$

Revista distribuida en las bases de datos:

LATINDEX, DOAJ, REDIB, IRESIE, CLASE, DIALNET, SHERPA/ROMEO, QUALIS-CAPES, MIAR

Revista registrada en los directorios:

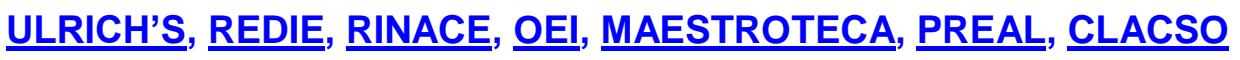




\title{
Adopción del modelo educativo por competencias, desde la categoría de Campus de Bourdieu
}

\author{
Adoption of the competence-based educative model, from the Campus category of Bourdieu
}

\section{Elveri Figueroa Escudero ${ }^{1}$ Verónica García Martínez²}

\begin{abstract}
Resumen: Se reporta la experiencia de la Universidad Popular de La Chontalpa, en Tabasco, México, en la cual se diseñó e implementó el Modelo de Educación por Competencias (MEC) en 11 de sus Programas de Formación Profesional (PFP) a partir de 2010. La investigación fue exploratoria y la metodología consistió en entrevistar en forma individual a 11 académicos que participaron en el proceso de rediseño curricular, desde un enfoque cualitativo. El objetivo fue ofrecer una explicación sociológica desde la perspectiva del campus de Bourdieu, que permita la reflexión de los distintos niveles de complejidad de una adopción institucional de este tipo de modelos. El uso del concepto en el análisis del fenómeno nos permite dilucidar las construcciones elaboradas por los agentes implicados en el entramado, que representa su propio campus desde la postura crítica de la sociología comprensiva. Los resultados muestran que en la adopción de nuevos modelos educativos se presentan rasgos de socialización distintos que impactan cuando menos, en dos ámbitos: la gobernanza institucional y el desarrollo de las funciones sustantivas de las organizaciones educativas. El peso político, económico y social de las agencias transnacionales y las nacionales, determina en cierto modo, la reproducción del modelo entre los agentes imbricados en el campus, pero, en cambio, se genera un desequilibrio de fuerzas en el interior de este, debido a la imposición de un modelo que no posee líneas orientadoras claras para su adopción.
\end{abstract}

Palabras clave: modelo educativo, competencia, diseño curricular, educación superior, México.

\begin{abstract}
This paper reports the experience of the Universidad Popular de La Chontalpa, in Tabasco, Mexico, in which there was designed and implemented the Competency Based Learning (CBL) in 11 of their professional training programs since 2010. This was a qualitative and exploratory research and the methodology consisted of individual interviews to 11 professors who participated in the process of redesigning the syllabus, from a qualitative approach. The objective is to offer a sociological explanation from a Bourdieu's Campus standpoint that will enable people to appreciate the different levels of complexity of adopting this kind of models in an institution. The use of the concept in the phenomenon analysis allows us to elucidate the constructions made by the agents involved in the system representing their own campus from a critical view of the comprehensive sociology. The results demonstrate that the adoption of new educational models have different socialization features, which to a certain extent have an impact in two areas: the institutional governance and the development of the substantive functions of educational organizations. The political, economic, and social impact of the transnational and national agencies determines to a certain degree the reproduction of the model among agents involved in the campus. However, the imposition of a model generate disparity at the interior of the campus due to the lack of clear guidelines for its adoption.
\end{abstract}

Key words: educative model, competence, curricular design, higher education, Mexico.

\footnotetext{
1 Profesor adscrito a la División de Ciencias Económico-Administrativo (DESCSA) de la Universidad Popular de La Chontalpa (UPCH). México. Maestro en Educación por la Universidad Juárez Autónoma de Tabasco (UJAT). Dirección electrónica: elveri.figueroa@gmail.com

2 Profesora-investigadora de la División Académica de Educación y Artes (DAEA) de la Universidad Juárez Autónoma de Tabasco (UJAT). México. Doctora en Ciencias Sociales por la Facultad Latinoamericana de Ciencias Sociales (Flacso). Dirección electrónica: veronica.garcia@ujat.mx
}

Artículo recibido: 15 de junio, 2016

Enviado a corrección: 8 de diciembre, 2016

Aprobado: 13 de febrero, 2017 


\section{Introducción}

La propuesta —en el discurso- de las agencias públicas ha impulsado la formación por competencias en las dos últimas décadas del siglo XX y en los primeros 15 años del tercer milenio. En los proyectos de la Organización de las Naciones Unidas para la Educación, la Ciencia y la Cultura (Unesco); así como en la Organización para la Cooperación y el Desarrollo Económico (OCDE), se trata de regular (de forma directa o indirecta) el sistema de educación superior; sobre todo al señalar la pertinencia de la adopción de modelos innovadores.

Son las agencias gubernamentales las que tienen un campus amplio de acción en la educación superior. Sus redes de comunicaciones son bastante diversificadas: algunas participan con préstamos económicos a los países, mayormente a las naciones en desarrollo. Otras fungen como foros de debates, con el propósito primordial de definir las perspectivas de investigación, el diseño de las políticas y la concreción de los proyectos. La mayoría se encarga de la conformación de los datos, estadísticas y del análisis relevante; con la intención de generar la literatura especializada, en formatos diferentes: reportes, periódicos, semanarios, informes, enciclopedias, ensayos, manuales, monografías o libros. Entre las temáticas emergentes tratadas están la evaluación, el aseguramiento de la calidad, acreditación o rendición de cuentas, por un lado. Por el otro, la ayuda financiera a los estudiantes, la planificación de la profesión académica y la innovación educativa; muchas veces, la definición de las políticas públicas tiene un enfoque internacional y pocas veces regional o nacional (Altbach, 2014).

Las acciones de las agencias gubernamentales son realizadas con fines ideológicospolíticos distintos, en el marco de una multiplicidad de proyectos y acuerdos, que tratan de dirigir sus esfuerzos hacia el desarrollo y cooperación internacional de la agenda pública mundial (Cobos, 2014). El resultado de la intervención de los organismos multilaterales, en la educación superior, ha consistido en cinco tareas principales: a) la definición de objetivos milenarios, b) el consenso de financiamiento e inversión educativa, c) la declaración de principios universales, d) el establecimiento de mecanismos de planificación y de control y e) los debates sobre la gobernanza académica (Delgadillo, Segura y Ayala, 2012).

En relación con la participación de las agencias supranacionales, Muñoz (2002, p. 106) destaca que "la promoción del cambio institucional es un objetivo común de los organismos internacionales, gobiernos y asociaciones de universidades". En este sentido, Ibarra (2001) sostiene que en torno a los establecimientos académicos, y en sentido estricto, existe un debate centrado en una probable empresarialización de las organizaciones académicas. El 
autor afirma que las universidades se han visto obligadas a "re-inventarse", aunque, para Miranda (2001, p. 34), esto significa el "neocorporativismo académico emergente".

Lo anterior hace suponer que el modelo educativo por competencias en los últimos años representa uno de los instrumentos principales utilizados para solucionar problemáticas relacionadas con los procesos de formación académica en la educación superior, tanto en México como en otros países de Latinoamérica. Su propósito principal es lograr la eficacia y eficiencia de los programas formativos, lo que a la postre contribuye al diseño de una arquitectura educativa sobre la que existen puntos de vista tanto a favor como en contra. Algunas estrategias incluidas en esta arquitectura repercuten no solo en la toma de decisiones de las autoridades universitarias, sino en los roles de profesores y estudiantes. Las más comunes de estas estrategias son el diseño de planes y programas formativos, la definición de perfiles profesionales, la formulación de proyectos y la actualización continua.

Que el modelo de educación por competencias esté presente en un gran número de IES latinoamericanas no es algo tan cuestionable, dada la tendencia hacia la implementación de este modelo en la educación superior. Lo que sí es digno de analizar es la forma particular en que los diseñadores curriculares de cada organización educativa entienden y aplican este enfoque, pero, sobre todo, las interpretaciones un tanto subjetivas que el claustro docente realiza en el ámbito de su aplicación; es decir, la forma en que se desarrolla el proceso de enseñanza y la manera en que se provoca el aprendizaje en el estudiantado.

Sobre la implementación del enfoque por competencias, se pueden encontrar varios casos en México; por ejemplo, la Universidad de Sonora (Unison), que en la reestructura de su modelo curricular y en la actualización de los planes de estudio consideró como eje primordial este enfoque (Unison, 2014). De igual forma, la Universidad Autónoma de Nuevo León (UANL), desde el 2008, diseñó su modelo educativo centrado en cinco ejes rectores, uno de ellos es la educación basada en competencias (UANL, 2012). Otra de las instituciones que fomenta el desarrollo de competencias profesionales y sociales es la Universidad Autónoma de Querétaro (UAQ, 2007). El modelo educativo de la Universidad de Guanajuato (UG) también promueve las competencias genéricas y específicas (UG, 2011). Asimismo, el modelo de la Universidad Juárez Autónoma de Tabasco (UJAT) tiene entre sus rasgos principales la definición de competencias profesionales (UJAT, 2012).

En este sentido, muchas de las IES han definido su perfil profesional a partir del modelo de educación por competencias. La Universidad Popular de La Chontalpa (UPCH), una institución pública estatal ubicada en el sur de México, es una muestra de ello. En el 2010, se 
reestructuraron los 11 programas académicos de formación profesional con criterios de competencias. Se centró la atención en el estudiante desde cuatro dimensiones: profesional, intelectual, humana y social. Se buscó la promoción del autoaprendizaje permanente, la construcción, deconstrucción y socialización del conocimiento relevante y pertinente, la formación ética centrada en los valores humanos y sociales, el desarrollo de habilidades de razonamiento y actitudes que permitan la aplicación de conocimientos teóricos en la dinámica de un contexto académico y laboral cada vez más internacionalizado.

En el 2011, se realizó una investigación para conocer la experiencia de los coordinadores sobre el proceso de implementación del modelo. El propósito del presente documento es elaborar una explicación sociológica sobre este proceso. Para tal fin se considera como base el punto de vista de los 11 académicos entrevistados durante el trabajo de campo, a fin de contrastar la evidencia empírica con el concepto de campus, como categoría teórica central de la sociología de Pierre Bourdieu (2013), debido a que encontramos coincidencias importantes entre los hallazgos empíricos de nuestra investigación y los elementos teóricos del concepto.

\section{Primera mirada conceptual: el Campus como forma de explicación microsocial}

El edificio teórico que construyó Bourdieu se orienta a tratar de explicar los fenómenos microsociales sobre la base de la reproducción de las prácticas cotidianas. Su trabajo no solo se basa en una actividad meramente filosófica o gnoseológica, sino en estudios empíricos como encuestas realizadas a más de 109563 franceses. Su análisis lo fundamentó al recoger hallazgos en, por lo menos, siete temáticas grandes: a) el tiempo libre y las prácticas culturales, b) la lectura de la prensa, c) los negocios, d) el tiempo libre en relación a las condiciones de vida, e) el consumo de las familias, f) la formación-cualificación profesional y g) los ingresos económicos (Bourdieu, 2013).

Pese a los estudios realizados a grandes masas, el enfoque de este autor lo podemos ubicar básicamente en el terreno microsocial, de ahí su atribución por una sociología estructural constructivista (Bourdieu, 2000). Este teórico se interesó por la vida cotidiana de los sujetos, sus relaciones interpersonales y estilo de vida dentro de las estructuras sociales. Sus aportaciones teóricas se evidencian a través de las categorías empíricas emergidas de su trabajo. Una de estas categorías, en la que nos hemos enfocado, es la de campus, la cual a partir de la segunda mitad del siglo XX se convierte en un tópico del análisis sociológico. 
Bourdieu y Wacquant (2012, pp. 134-135) teorizaron al campus en los términos que se indican:

Una red o configuración de relaciones objetivas entre posiciones. Estas posiciones están objetivamente definidas, en su existencia y en las determinaciones que imponen sobre sus ocupantes, agentes o instituciones, por su situación presente y potencial (situs) en la estructura de distribución de especies del poder (o capital) cuya posesión ordena el acceso a ventajas específicas que están en juego en el campo, así como por su relación objetiva con otras posiciones (dominación, subordinación, homología,...).

Los rasgos que Bourdieu (2012) le atribuyó al campus son cuatro: a) existe, entre los participantes, una lucha constante, propósitos y estrategias, que son distintos entre sí; b) se siguen regularidades y reglas que no siempre son explícitas ni están codificadas; c) los participantes buscan imponer los principios de jerarquización más favorables para sus propios intereses que, de manera individual o colectiva, pretenden salvaguardar o mejorar su posición y d) las relaciones de fuerzas definen la estructura del campo. Cierta similitud tiene la definición de Giddens (2010, p. 891) en relación con este concepto:

Los diferentes lugares sociales o foros en los que tienen lugar las luchas competitivas basadas en las formas de capital. Es a través de estos campos como se organiza la vida social y actúan las relaciones de poder, y cada uno tiene sus propias 'reglas de juego'.

El concepto sustancialmente se distingue por poseer algunos elementos que lo particularizan y algunas causas que lo originan, estos son de organización, intervención, distribución, arreglo y coerción (Tabla 1). 
Tabla 1: elementos del campus: sus rasgos identitarios

\begin{tabular}{|c|c|c|}
\hline Elementos & Descripción & $\begin{array}{c}\text { Causas que originan la presencia de } \\
\text { cada elemento }\end{array}$ \\
\hline Organización & Estructura distributiva de capitales. & $\begin{array}{l}\text { Producción o negociación de distintas } \\
\text { clases de capitales. }\end{array}$ \\
\hline Intervención & $\begin{array}{l}\text { Participación con conocimiento de } \\
\text { estrategias propias de cada espacio en } \\
\text { particular. }\end{array}$ & $\begin{array}{l}\text { Interés de los agentes o instituciones en } \\
\text { participar. }\end{array}$ \\
\hline Distribución & $\begin{array}{l}\text { Diferentes posiciones de los agentes } \\
\text { (productores o consumidores) o } \\
\text { instituciones. }\end{array}$ & La disputa de los capitales. \\
\hline Arreglo & $\begin{array}{l}\text { Configuración de relaciones de alianza o } \\
\text { conflicto, de competencia o de } \\
\text { cooperación. }\end{array}$ & $\begin{array}{l}\text { Mantenimiento del orden social a través } \\
\text { de la interiorización de las condiciones } \\
\text { sociales y económicas. }\end{array}$ \\
\hline Coerción & $\begin{array}{llll}\text { Imposición } & \text { de } & \text { reglas por } & \text { los } \\
\text { dominantes, } & \text { que } & \text { convienen a } & \text { sus } \\
\text { intereses. } & & & \end{array}$ & $\begin{array}{l}\text { La apropiación de bienes o recursos } \\
\text { económicos, culturales y sociales en } \\
\text { juego que determinan la entrada y la } \\
\text { permanencia. }\end{array}$ \\
\hline
\end{tabular}

Fuente: Elaboración propia con información tomada de Bourdieu (1973, citado en Castón, 1996) y Bourdieu (1971, 1983, 1984 y 1995; citado en Velasco, 1998).

Cada campus, como espacio estructurado de posiciones, posee reglas y políticas propias que le determinan la posición objetiva o no a los agentes o instituciones participantes. Además, de que el campus funciona con leyes generales invariables que actúan como mecanismos universales, donde existen categorías de intereses específicos y objetos en juego (Bourdieu, 2003). Los campos son espacios de lucha competitiva en los que se utilizan una variedad de recursos (tipos de capital). En este modelo se considera que la estructura y la agencia están íntimamente relacionadas y no son opuestas (Giddens y Sutton, 2015). Este concepto ha sido analizado tanto en sentido teórico como en el empírico. Kurt Lewin (1978) profundizó en el concepto de campo (al que Bourdieu llamó campus) desde la Psicología, usó esta noción para elaborar su propuesta teórica de campo psicológico. En ella, Lewin aclaró que el campo ejerce influencias en la conducta individual y grupal. Para él, la conducta particular es una función de las personas y de su ambiente y solo a partir de los individuos se construye un campo específico. También, esclareció que el campo es determinante en la estructura de un grupo, pues le imprime rasgos que los distingue de otras colectividades (Fernández y Puente, 2009).

El concepto de campus, también, inspiró a los neoinstitucionalistas para estudiar las estructuras internas y externas de las organizaciones. A Powell y a Dimaggio les interesó analizar "la estructura de los ambientes, los efectos de los macro y microniveles, y la autonomía analítica de las macroestructuras" (Powell y Dimaggio, 2001, p. 106). Para estos 
autores, la existencia de un campo en específico está determinada por una estructuración institucional, la cual consta de cuatro etapas: a) interacción interorganizacional, b) aparición de patrones de coalición y de dominio, c) aumento de información y d) desarrollo de la conciencia entre los participantes.

La categoría teórica del campus, también, fue utilizada para comprender y explicar los procesos formativos. En lo particular, se estudió la trayectoria académica de estudiantes de posgrado de la Universidad Nacional Autónoma de México (UNAM). Con el apoyo del concepto, se explicaron los factores más preponderantes que incidieron en cuatro procesos interrelacionados del trayecto académico de 24 estudiantes egresados: la incorporación, socialización, tutoría y graduación. La investigación demostró con insumos empíricos que el campus, "se halla en medio de luchas y alianzas por el dominio del poder simbólico de nominar lo que es legítimo en la formación de estudiantes, la producción de investigaciones y de tesis" (Sánchez-Dromundo, 2007, p. 7).

Se desea exponer la adopción del modelo de educación por competencias en la UPCH desde la propuesta de Pierre Bourdieu. Para eso, primero se presenta el basamento metodológico del estudio original; y segundo, mostramos un análisis de la aplicación del modelo con la ayuda de los componentes teóricos del campus.

\section{Metodología}

El propósito es explicar la forma en que se adoptó el modelo de educación por competencias en la UPCH. El interés en el 2011 fue indagar —entre otras aristas- sobre las circunstancias que propiciaron la innovación académica, se identificaron las etapas, los obstáculos y retos institucionales presentes. En el discurso de los informantes constatamos que, para la mayoría de ellos, la implementación los tomó por sorpresa debido, principalmente, a la adopción de un plan con criterios de flexibilidad curricular que apenas llevaba unos cuantos años y que no había sido evaluado para cambiarlo por otro distinto. Aunado a ello, el profesorado sentía incertidumbre sobre el efecto que provocaría la transición curricular en las actividades académicas, así como el tipo de comportamiento y trabajo que se le solicitaría. Aparte de esto, los administradores universitarios desconocían sobre la gobernabilidad académica, para el desarrollo de la gestión, extensión y vinculación institucional, bajo un esquema de educación diferente.

Se diseñó —en el 2011- un guion de entrevistas con 23 ítems agrupados con tres categorías analíticas. La primera, se denominó metodología de la implementación; la segunda, 
obstáculos, y la tercera, retos; fundada principalmente en los objetivos del estudio. En agosto y septiembre de 2011, se realizaron las entrevistas individuales a los 11 académicos que intervinieron en las diversas etapas del diseño y desarrollo (adopción) del modelo.

En el presente artículo, partimos del supuesto de que la adopción de modelos académicos se realiza en un conjunto de relaciones entre agentes diversos que intervienen según su capacidad de participación. Hecho que impacta al interior de las instituciones donde se presentan rasgos de socialización distintos traducidos en definiciones diferenciadas sobre el tipo de gobernanza institucional. Eso por un lado; por el otro, las actividades de docencia, investigación, difusión y extensión toman cada vez un rumbo diferente, más amplio. Los objetivos académicos de las instituciones de todos los niveles de enseñanza, pero, sobre todo la superior, son determinados externamente y moldeados para responder a propuestas específicas de organismos supranacionales.

En este reporte, se busca responder a la pregunta: ¿cómo se explica el proceso de implementación del modelo de educación por competencias en la Universidad Popular de La Chontalpa, desde la teoría del campus de Bourdieu? El diseño fue cualitativo y exploratorio. Es cualitativo porque el objetivo primordial fue documentar e interpretar el caso institucional que era familiar y esta metodología ayudó a reconstruir el proceso curricular, y exploratorio porque representó el primer acercamiento al fenómeno educativo. También, es inductivo, caracterizado por la recolección de las experiencias y significados particulares de los actores insertos en las estructuras institucionales. (García, González y Ballesteros, 2001) A partir de la interpretación de estas experiencias, se buscó analizar estos resultados desde la teoría de campus a través de un proceso hermenéutico o por lo menos exegético.

La articulación del paradigma cualitativo y la metodología exploratoria consistió formalmente, en cinco etapas que se sintetizan de la manera que se describe.

Primera, en términos de sociología descriptiva, podemos afirmar que la didáctica práctica de la investigación consistió en explicar el cambio curricular en forma sintética y clara y sirvió para analizar indicadores analíticos. Se nos permitió observar el cambio en el sistema universitario, la metodología y las implicaciones de las competencias en la educación universitaria en México. En la segunda, analizar las características principales del campus, que consistió en observar la forma en que se desarrollaron los procesos, operaciones y mecanismos del cambio curricular universitario. Bourdieu realizó estudios empíricos en siete categorías, en las cuales puso su atención en las relaciones interpersonales de los sujetos. En 
ese sentido, explicó que el campus es una red definida de relaciones entre las personas y las instituciones u organizaciones.

Luego, en la tercera etapa en específico, la metodología exploratoria nos sirvió para conocer las decisiones, un tanto estratégicas, que los directivos tomaron en torno a la forma en que se desarrolló el espacio interno de la universidad. Lo que contribuyó en la interpretación del cambio del currículum. En la cuarta, el desarrollo curricular nos permitió identificar principios y dimensiones de innovación académica, que definieron una imagen institucional universitaria. Posteriormente, hemos identificado los efectos del cambio curricular. Por último, se distinguieron las implicaciones para la implementación de un modelo de educación basado en competencias. El recorrido sobre el proceso de implementación sirvió para documentar el proceso de implantación y la organización del trabajo académico. Es decir, con este trabajo recopilamos el desarrollo, dinámica y relaciones laborales; iniciadas con la forma de trabajo nueva. Además, se definió la misión y visión para la educación superior en el siglo XXI, en el caso estudiado. También, se definieron estrategias programáticas del modelo curricular implementado.

A partir de lo anterior, se contó con la posibilidad de establecer una relación de las preguntas de investigación con la perspectiva de estudio del caso interpretativo, para definir la situación de investigación y lo que allí ocurría. De hecho, la perspectiva interpretativa permitió observar la forma en qué se produjo cada etapa del diseño curricular. Durante el proceso de investigación, se distinguieron dos formas de gobernanza: a) la de tipo vertical, es decir, la forma en que los directivos académicos administraron al grupo de diseñadores curriculares y b) el desarrollo de una conducción horizontal del proceso, la forma en que los responsables de la elaboración de cada plan y programas de estudio académico se apoyaron entre ellos. Finalmente, pudimos conocer la naturaleza de la experiencia de trabajo del equipo y de comprender la diversidad de perspectiva de los entrevistados.

\section{Resultados y análisis}

En este apartado, se presenta el cuerpo teórico del campus que fundamenta nuestro trabajo, seleccionado de la diversidad conceptual de Pierre Bourdieu. Se aplica al análisis de la adopción del modelo de educación por competencias, a partir de la revisión del caso institucional abordado. La decisión de análisis desde la propuesta teórica, de este autor en términos generales, es la necesidad de comprender el proceso institucional de la 
implementación desde el punto de vista de los actores (académicos) individuales, insertos en una estructura de autoridad.

En este documento únicamente se aborda y discute el concepto "bourdieusiano" de campus. Varias son las razones que orientan este análisis: primera, porque reporta en conjunto una claridad teórica que ayuda a la comprensión sobre el rumbo del cambio institucional. Segunda, permite reconocer que un proceso de transición curricular por naturaleza es complejo por las distintas trayectorias de los agentes y por la propia dinámica de los procesos institucionales y a veces estos dibujan o desdibujan los objetivos académicos. La tercera de estas razones es la oportunidad que aporta este concepto para explicar, según criterio propio, la intención por la cual en los establecimientos de educación terciaria se realizan innovaciones centradas en las competencias. La cuarta razón es que ayuda a asumir una postura reflexiva sobre cierta resistencia natural que manifiesta el claustro de profesores desde el 2010 a la fecha. La quinta es para dar un orden lógico y aplicar el sentido común sobre la notoria y pesada influencia de las agencias públicas en el diseño institucional y a veces las tan impositivas políticas educativas que estuvieron presentes en la fundamentación de cada uno de los 11 planes de formación profesional. La sexta, para implementaciones o reestructuras curriculares futuras en el caso que referimos, sirve como retroalimentación para reevaluar el proceso y sugerir a los decisores académicos que se consideren los componentes principales del proceso de enseñanza y aprendizaje.

La intención de considerar al concepto de campus obedece a una decisión analítica porque este es, considerado por Bourdieu (2001), como un círculo hermenéutico que implica configuraciones de los distintos tipos de capitales, de interés de los agentes, determinado por ciertos rasgos de adscripción familiar, educativa o social. Es decir, es estimado como un espacio multidimensional integrado por una constelación de variados componentes en su interior.

Bourdieu define al campus (microcosmos sociales) como el agrupamiento de relaciones de fuerzas entre agentes o instituciones, en la lucha por formas específicas de dominio y monopolio de un tipo de capital eficiente en él. Este espacio se caracteriza por relaciones de alianza entre los miembros, en una búsqueda por obtener mayor beneficio e imponer como legítimo aquello que los define como grupo; así como la confrontación de grupos y sujetos en la búsqueda por mejorar posiciones o excluir grupos. Además, es un universo en el que las características de los productores están definidas por su posición en las relaciones de producción, por el lugar que ocupan en un determinado espacio de relaciones objetivas 
(Bourdieu, 1980). El espacio social del campus es "la realidad primera y última, puesto que sigue ordenándolas representaciones que los agentes sociales puedan tener de él” (Bourdieu, 1980, p. 23).

En relación con este concepto, el aporte principal de Bourdieu ha sido, cuando menos, en dos sentidos. El primero implica que los agentes (o grupos de agentes) actúan y que, como resultado, producen efectos desde y para el campus en el que participan. El segundo, que la participación de los agentes o clases de agentes estará determinada por el tipo de capital que opera en el campus y de la familiaridad que tengan los agentes con este. Así, se señala que la distribución de estos al interior de cada campus puede ser explicada desde el mismo estado y proceso de constitución de las estructuras de distribución de los agentes propios (Bourdieu, 2002).

Las estructuras y las fuerzas entre sí de un campus determinan en gran parte su dinámica y complejidad. Aquí, los agentes están ubicados en posiciones diferentes; los lugares de ellos dependen del capital del que dispongan y de la percepción que tengan sobre el espacio que ocupan. Existen agentes que dominan y ellos hacen que el campus funcione a su conveniencia, a pesar de que se enfrenten a obstáculos, resistencias y retos, lo que provoca procesos de producción y reproducción de desequilibrios y asimetrías en las prácticas (Bourdieu y Wacquant, 2012).

Es en relación con las prácticas que se realizan en un campus determinado por lo que Bourdieu planteó un formulario sociológico:

...la unidad que se disimula bajo la diversidad y la multiplicidad del conjunto de unas prácticas realizadas en campos dotados de lógicas diferentes, luego capaces de imponer unas formas de realización diferentes (de acuerdo con la fórmula: [(hábitus) (capital)] + campo = práctica). (Bourdieu, 2013, p. 115)

Por eso, se pretende delinear un mapa institucional-sociológico sobre la implementación del modelo de educación por competencias en la UPCH. Con este propósito, hemos organizado el análisis en tres dimensiones: a) el peso de las agencias públicas sobre la universidad pública estatal, b) la relación entre las políticas educativas de las agencias públicas y las acciones prácticas de los establecimientos académicos poco consolidados y c) la configuración de las relaciones del trabajo académico, a partir de la adopción del modelo de educación por competencias (Figura 1). 
Figura 1: el trabajo académico en la Universidad Popular de La Chontalpa: su desarrollo, dinámica y relaciones laborales

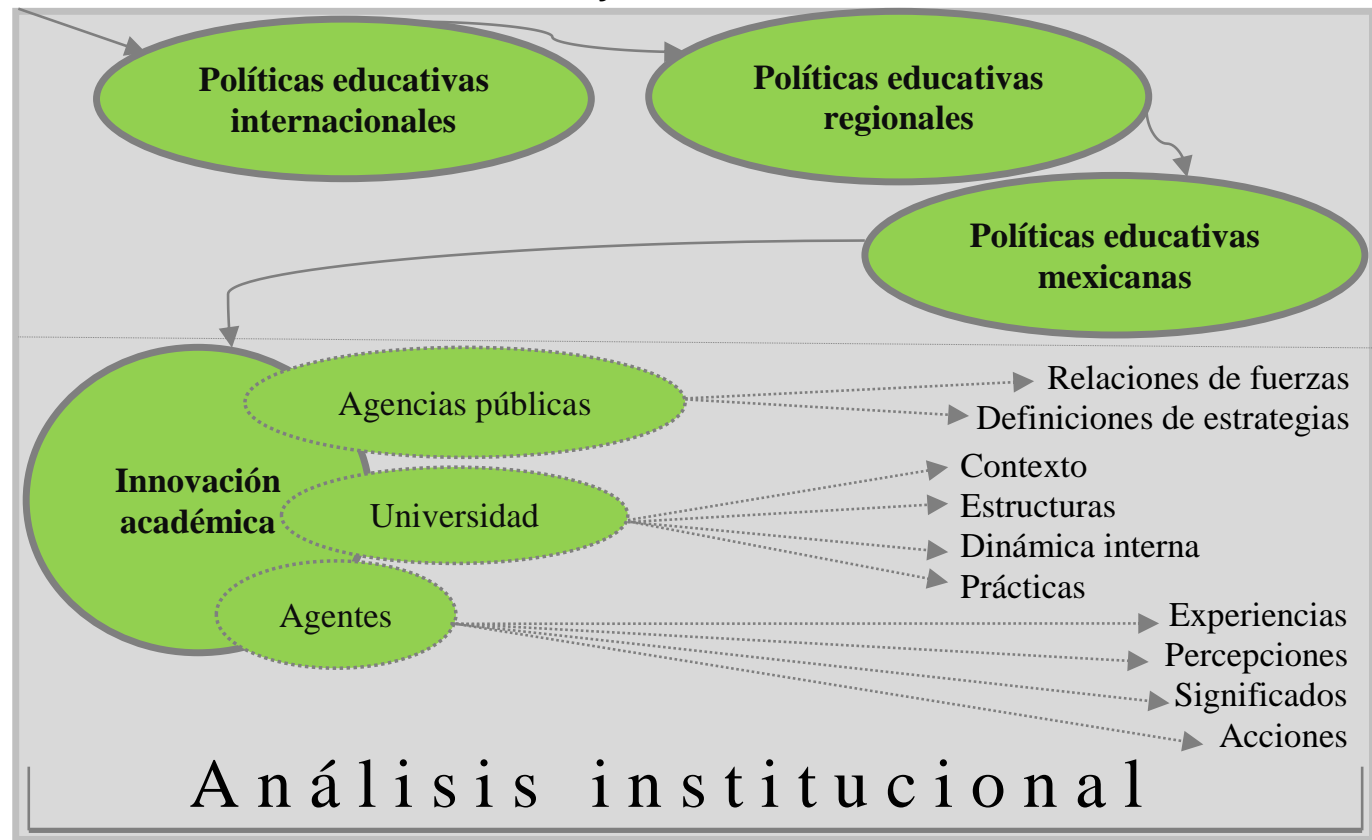

Fuente: Elaboración propia, sobre la base de la interpretación de los resultados.

Esta parte del análisis tiene como propósito fundamental exponer los rasgos particulares que distinguen el trabajo académico de una institución mexicana; que ha realizado cambios sustanciales en su modelo educativo en dos ocasiones, con el fin de mostrar elementos que permitan explicar el desarrollo, dinámica y prácticas institucionales con elementos teóricos abordados en este estudio.

a) El peso de las agencias públicas sobre la universidad pública estatal: se observó que el espacio principal -el campus - en el estudio que referimos estuvo determinado, primero, por la aplicación de una política educativa en forma unidireccional principalmente, por las autoridades universitarias en turno. Segundo, por una planeación que tuvo como propósito fundamental el progreso académico, aunque determinado por las reglas del juego de un perfil educativo en específico, impulsado y modelado por instancias a veces educativas, pero también, económicas; de modo que diversidad de propósitos adicionales a los académicos juegan en esta implementación. En este sentido subyacen las estrategias de reproducción (de las agencias), que tienden a perpetuar la estructura social (Bourdieu, 2002).

Son cuatro los componentes con los que cuentan estas agencias internacionales, que les permiten, hasta cierto punto, una participación mayoritaria en la definición de las políticas de la educación superior. El primero consiste en la presencia geográfica que ocupan. Esto es, 
sus sedes, centros u oficinas regionales o subregionales ubicadas estratégicamente, donde se les brinda la oportunidad de conocer con profundidad las necesidades educativas de cada país. El segundo es el poder económico que ostentan en virtud que son frecuentemente las proveedoras de préstamos y becas, para que las instituciones de educación superior realicen sus funciones académicas de forma más diversificada y con mayor impacto social. El tercero es el poder de convocatoria que tienen, a través de las rondas, conferencias, convenciones, foros o reuniones interministeriales, de alcances regional, internacional o mundial. Esto se aprecia en los formatos de sus eventos, que representan espacios donde se presentan estudios, indicadores y estadísticas como las fuerzas más preponderantes que moldean y constriñen al campus académico y que a través de los discursos son legitimados y asumidos como referentes poco cuestionables. El cuarto componente está centrado en el interés por la realización de estudios e investigaciones. Las agencias internacionales son las encargadas de formular propuestas, metodologías y enfoques, de definir líneas de análisis, de determinar retos y de establecer prioridades, de diseñar escenarios y construir las tendencias. Esto a partir de los estudios e informes temáticos de gran envergadura, de los cuales son responsables de administrar los financiamientos, diseño, realización y coordinación e incluso, se encargan de calendarizar los objetivos de desarrollo del primer siglo del tercer milenio.

Tal parece que las agencias públicas de orden transnacional y, subsecuentemente, las mexicanas en ocasiones diseñan políticas educativas con lógicas distintas. El proyecto de la Unesco sobre el perfil de las competencias se centra primordialmente en el desarrollo social y humano. Para la Unesco resulta imprescindible la promoción de: a) conocimientos generales y b) contenidos valorables. En el caso de la OCDE y el Banco Mundial (BM), estos promueven la formación de los recursos humanos, la mejora de la capacidad productiva y la calidad del trabajo. Estos últimos apuestan sobre las bases de la teoría del capital humano (TCH), lo que, a nuestro parecer, impone determinadas formas en la realización de las funciones académicas.

Estos componentes son solo una explicación parcial de la justificación que tienen, a semejanza de "los pasaportes", los organismos internacionales para introducirse e imponerse ante los establecimientos académicos, independientemente de la apropiación hecha por los agentes locales (Bourdieu, 2013). Por un lado, su presencia se ve reforzada por los gobiernos de naciones industrializadas; por el otro, por las universidades con mayor presencia académica, dedicadas a la producción de investigaciones. Esto genera una asimetría con las universidades que, con dificultades, solo se dedican a la enseñanza como es el caso de la UPCH, cuya presencia en el ámbito investigativo es reducida. 
El efecto perverso más significativo —y a menudo más imprevisto—al parecer es que la desigualdad académica que generan los organismos internacionales produce desequilibrios institucionales. Sobre todo porque el trayecto que recorren las políticas educativas desde el epicentro de las agencias internacionales hasta su ámbito de aplicación es bastante largo y dificultoso. En este camino intervienen diferentes niveles de mando gubernamentales y administrativos, por una parte; por la otra, los distintos actores participantes realizan reformas a las políticas, muchas veces con sesgos que distan en gran medida de los propósitos originales. La situación se agrava cuando las organizaciones educativas no cuentan con una estructura académica consolidada. Entonces, existen pocas barreras para que las agencias internacionales introduzcan sus procesos, metodologías y enfoques de trabajo.

Frente a esto valga la pregunta, ¿cuál sería la forma más apropiada de equilibrar las fuerzas de organización, intervención, distribución y coerción de las agencias internacionales en la determinación de las estrategias académicas en las Universidades Públicas Estatales con Apoyo Solidario (Upeas), del sureste mexicano? En la medida en que las universidades se convierten únicamente en transmisoras y reproductoras de conocimientos, la posibilidad de que la lógica y estrategias de las agencias internacionales se introduzcan en ellas es bastante significativa y las dinámicas y procesos internos se verán afectados. Por eso, las universidades deben centrar sus esfuerzos en la definición de políticas institucionales estratégicas que atiendan a una misión cuatripartita: enseñanza, investigación, difusión y extensión; funciones que deben definir roles más abarcadores del quehacer académico para el claustro del profesorado. Asimismo, las actividades de gestión que realiza el cuerpo administrativo y las funciones de gobernabilidad, pero también de gobernanza que deciden los funcionarios universitarios y los cuerpos docentes. Otra forma de equilibrar las fuerzas es, simplemente, que en la medida que las políticas internacionales definidas por las agencias supranacionales sean compatibles con las características económicas, culturales, sociales y educativas de la región; en esa medida las universidades estatales tendrán una participación más representativa y se lograría el cierre de brechas; es decir, se podría reducir las diferencias existentes en los indicadores académicos.

\section{b) La relación entre las políticas educativas de las agencias públicas y las acciones} prácticas de los establecimientos académicos poco consolidados: para el diagnóstico de factibilidad y pertinencia académica de los planes de estudio, con el fin de lograr una reestructuración curricular eficaz y eficiente, se consideraron las recomendaciones de 
agencias públicas en primer lugar. En segundo, la revisión de documentos nacionales y estatales; lo que comprendió el registro, en un inicio, de medidas e indicadores de la Organización de las Naciones Unidas para la Educación, la Ciencia y la Cultura (Unesco); de la Organización para la Cooperación y Desarrollo Económico (OCDE) y del Banco Mundial (BM). Para después, realizar una revisión del Plan Nacional de Desarrollo de México (PND) y del Programa Nacional de Educación mexicano (PNE). Sucesivamente, se consideró el Plan Estatal de Desarrollo de Tabasco (PED) y el Programa Estatal de Educación (PEE). Luego, se analizó el Plan Municipal de Desarrollo (PMD); y finalmente, el Plan de Desarrollo Institucional de la UPCH (PDI).

Sin embargo, ¿de qué manera esta imposición trastoca la gobernanza institucional? Un análisis de la información recuperada en el trabajo de campo nos permite afirmar que en las políticas educativas se ha creado toda una industria sobre las innovaciones en la educación superior, lo que finalmente les permite a las universidades "pequeñas" que se vean en la necesidad de "copiar" los desarrollos científicos y tecnológicos del exterior (agentes: "tendencias de la educación, principalmente...", "obedeció a los cambios que se han presentado a nivel mundial y nacional en torno al proceso de enseñanza y aprendizaje que...", "considerar el trabajo de otras universidades..."). Esto tal vez contribuye a que no se defina una visión educativa explícita, con posibilidad de formación realista.

No obstante, las presiones no solo fueron externas, sino también internas. Las autoridades académicas de ese entonces decidieron implementar un nuevo modelo educativo, tanto para legitimar su ascenso y permanencia en los puestos administrativos, como para hacerle saber a la comunidad universitaria que era necesaria la innovación educativa. Esta innovación se entendió en un sentido muy acotado, pues se aseguró que se requería un cambio en el proceso de enseñanza y aprendizaje (agentes: "estar a la par con otras universidades del mundo...", "incursionar en el nuevo ejercicio pedagógico", "tratar de beneficiar con un nuevo modelo y una nueva forma de enseñanza a los alumnos..."), algo que a primera vista parece obvio. Sin embargo, no lo es tanto si tomamos en cuenta que la innovación debe significar una vinculación intrínseca entre la universidad, la ciencia, la tecnología y la sociedad; y si agregamos que la infraestructura académica existente no permite en forma eficaz y eficiente un vínculo fuerte con otras universidades, institutos, centros de investigación, empresas e industrias; el problema se vuelve denso.

El peso político, económico y social de los organismos públicos permite impulsar políticas y recomendaciones para que las instituciones académicas adopten ciertos criterios 
en la formación profesional. Al ser estos un medio organizativo del espacio público, crean un entorno estructurado que les permite poseer el monopolio sobre el diagnóstico y diseño de las políticas públicas en términos generales y de las educativas, en particular (macro campus). Son ellos los que se encargan de la conformación de los bancos de datos y del diseño de macroproyectos. Ciertamente, las políticas educativas que se diseñan determinan el rumbo nacional; sin embargo, estas debieran ser políticas diversificadas y no universales, que homologan los contextos que difieren en aspectos diversos (meso y micro campus).

El campus, debe ser entendido como una construcción sociológica y social, por dos razones: a) sigue reglas o regularidades y b) la participación de los actores, aumenta 0 disminuye su capital (en este caso institucional). El efecto principal que se tuvo con la implementación del modelo de educación por competencias fue la sorpresa, porque los actores no se lo esperaban, a pesar de las regularidades que el contexto dictaba. Los académicos, según la percepción de los administrativos entrevistados, participan en el desarrollo del modelo en mayor o menor medida, según las condiciones institucionales y de formación profesional se los permita. Visto así, pareciera que la universidad en estudio está atenta a los dictados de las corporaciones internacionales, más que a las propias necesidades de la comunidad docente o estudiantil, en virtud de lo cual el capital es inestable, y la construcción del campus.

Un comentario que parece interesante y que pone de manifiesto algunos rasgos relevantes sobre la adopción del modelo es el que se señala:

"Hubo desinterés por parte de algunas personas. Se presentaron problemas de resistencias al cambio y no querían participar en la elaboración. Dado que no existía experiencia en la implementación de modelos por competencias, costó demasiado trabajo coordinar todo el proceso y hacer que los maestros participaran”. (Comunicación personal)

\section{c) Configuración de las relaciones del trabajo académico, a partir de la adopción del} modelo de educación por competencias: más concretamente, la innovación institucional implicó nuevas dinámicas y prácticas de trabajo; lo que se tradujo en un cambio institucional, entendido este como la reorganización de los procesos académicos, administrativos y de gobernabilidad que se gestan a partir de factores externos e internos con sentidos y magnitudes diferentes, que provocan y condicionan las relaciones de los actores estratégicos (estudiantes, profesores, personal administrativo y funcionarios); que muchas veces son relaciones de contradicción, tensión y conflicto; con efectos generales un tanto difusos; o bien, 
pueden ser relaciones institucionales estratégicas que ayudan en el logro de los objetivos académicos, lo que se expresa en el grado de consolidación de la institución.

Entonces, ¿qué tipo de relaciones de trabajo y de prácticas académicas se introdujeron en la dinámica institucional de la Universidad Popular de La Chontalpa, con la innovación curricular de 2010? Para esto, se organizó el análisis centrado en el trabajo académico del profesorado (hábitus). Por eso, en el diseño del modelo, se demandan rasgos académicos y profesionales muy delineados del personal docente: a) un claustro de profesores que estén en la búsqueda constante de conocimientos nuevos; b) un perfil profesional acorde con la formación disciplinaria que pretenda el programa de formación profesional; c) habilidades pedagógicas para la instrucción básica y avanzada y d) el realizar investigaciones acordes a su campo disciplinario para nutrir los contenidos de las asignaturas que enseñan y diseño de materiales didácticos que sean del interés de los estudiantes.

Sin embargo, ¿cómo explicar las acciones de resistencia del personal docente, para el desarrollo del modelo académico? En medida distinta y según el testimonio de nuestros entrevistados, los profesores, en un porcentaje alto, no realizan una planeación académica estructurada de las asignaturas que les toca impartir. Algunos acotan el contenido que deben enseñar, lo que significa que no se cumple cabalmente con la atención al estudiante. También, la incongruencia entre los requerimientos académicos del modelo y el desempeño del plantel docente en el aula, es otra de las acciones de resistencia a las que se alude. Los docentes sienten la presión de actualizarse en sus respectivas disciplinas, pero requieren, en términos generales, de un sistema de incentivos más claro y justo. Es decir, que se evalúe su desempeño académico, se realice la promoción laboral, basada en criterios académicos y de competitividad, que logre sobreponerse al favoritismo de los funcionarios en turno. En este sentido, se dan lo que Bourdieu llama luchas entre "pretendientes y dominantes", una característica propia de los campos (Bourdieu, 1990) y una invariante, porque determina lo que está en juego, en este caso, la imposición de un modelo curricular.

Del trabajo académico que realizan los profesores existen experiencias diversas, según sea la naturaleza del campo disciplinario en donde participen. Algunos perciben que la operatividad del modelo fue difícil en los primeros años de su implementación y gradualmente se está en posibilidad de asimilar los procesos de reforma curricular. Los implicados afirman que el trabajo docente se complica por el hecho de tener la responsabilidad de cumplir con la definición y aplicación de metodologías que promuevan el desarrollo de competencias específicas en el estudiantado. Sienten que es necesario contar con una infraestructura 
académica sólida que facilite la consolidación del modelo académico implementado. No obstante, en la medida que el personal docente asimila la dinámica interna y las prácticas académicas, como actores situados dentro de una macroestructura de relaciones de poder, les atribuyen diversos significados e interpretaciones a las innovaciones académicas. (Agentes: “...sobre todo donde más problema tengo es en el área de las ciencias exactas, de las Matemáticas, por ejemplo, ellos consideran que...", "el reto más grande como docente ha sido el tener que desarrollar algunas metodologías y que el alumno las comprenda para que..." y "el nuevo modelo para evaluar da mucho trabajo, dado que se tiene que hacer como lo establece el modelo y exige más trabajo de parte del maestro").

El supuesto general que sostenemos, en este caso, es que el cambio institucional ha sido el resultado de las relaciones de fuerzas que, por un lado, impulsan la adopción de políticas educativas externas; por el otro, el cambio institucional, también, lo desarrollan las interacciones de los agentes académicos. Ellos actúan dentro de estructuras de dominación configuradas, lo que define sus prácticas institucionales en el ámbito universitario. Un campo, señala Bourdieu, se identifica (entre otras cosas) al definir aquello que está en juego y los intereses específicos que son irreductibles a lo que se encuentra en juego en otros campos y para que funcione este, es necesario que haya algo en juego y gente dispuesta a jugar, que esté dotada de los hábitus que implican el conocimiento y reconocimiento de las leyes inmanentes al juego, de lo que está en juego. En este sentido, se juega la permanencia en un sistema educativo global que los organismos internacionales dictan y los agentes aceptan las reglas del juego ante una inminente entrada a la modernidad educativa a través de la “innovación”, que no supone, sin embargo, muchas veces un ejercicio reflexivo de los agentes, sino, más bien, uno reproductivo.

\section{Conclusiones}

Aunque existe una distancia considerable para aportar hallazgos empíricos suficientes sobre la teoría sociológica de Pierre Bourdieu, es precisamente este corpus teórico el que nos ha permitido comprender las razones por las que en la educación superior mexicana, desde la postrimería del siglo XX y el inicio del tercer milenio, se adopta cada vez con mayor impulso la formación por competencias.

Desde el punto de vista de Bourdieu, todo sistema de enseñanza institucionalizado debe las características específicas de su estructura y de su funcionamiento al hecho de que le es necesario producir y reproducir, por los medios propios de la institución, las condiciones 
institucionales cuya existencia y persistencia (autorreproducción de la institución) son necesarias tanto para el ejercicio de su función propia de inculcación como para la realización de su función de producción de una arbitrariedad cultural de la que no es el productor (reproducción cultural). Tal reproducción contribuye a la reproducción de las relaciones entre los grupos o las clases (reproducción social).

En el hecho institucional de la UPCH, el rostro más visible del modelo estuvo integrado por los diversos planos que se le definieron: el socioeconómico, epistemológico, pedagógico y psicológico. Los académicos expresaron que el diseño buscó responder a los requerimientos regionales primeramente, después, a los nacionales. Cabe observar que en el diseño de los programas académicos se refleja un interés por atender las diversas problemáticas de los sectores productivos, aunque fue un tanto complicado para los profesores integrar los planes de estudio.

Según algunos de los relatos escuchados en las entrevistas, hubo por parte de la mayoría de los actores institucionales un consentimiento de aceptación y reproducción, porque tal vez desconocían que ello implica dominación. En este sentido, naturalizaron el peso de los factores externos, dado que la decisión de implementar el modelo se tomó sin que a ellos como responsables directos en cada uno de sus programas de estudio- se les hiciera saber. Incluso, fue una decisión que escapó al control de los académicos de mandos superiores. De ahí que no tuvieran la oportunidad de opinar, de ser escuchados, de consensar y analizar los posibles efectos que tendría definir la enseñanza por una parte, y el aprendizaje, por la otra.

Un aspecto más que se indagó en las entrevistas fue sobre las circunstancias que, según el cuadro de académicos elegidos, propiciaron la implementación del modelo por competencias en la UPCH. Ellos señalaron que el hecho de haberse adoptado el enfoque por competencias estuvo promovido, como ya señalamos, por distintos instrumentos de políticas que tienen un impacto significativo en el entretejido educativo. La verdadera posición dominante recae en quien en definitiva ejerce la acción.

El modelo de educación por competencias se impone como un esquema educativo que debe adoptarse en la enseñanza, sin considerar los rasgos particulares de cada estado nacional y de las instituciones. Lo que es aún más destacable es el hecho de que en otros países - por supuesto, los industrializados - ya han reorientado sus sistemas educativos en otros sentidos, puesto que el enfoque no resolvió las carencias en el aprendizaje. Si nos acercamos a una explicación sociológica, se diría que en el campus educativo se ha tendido a reproducir un mensaje unidireccional de la empresa a las instituciones educativas. Estas 
últimas se han visto obligadas a redefinir sus funciones sustanciales, lo que nos hace preguntarnos: ¿hasta qué punto es conveniente atender a los requerimientos y transformaciones empresariales?

En las recomendaciones que las agencias públicas han realizado — de forma colectiva— a las organizaciones educativas sobre las aparentes razones válidas para implementar el enfoque de las competencias en los proyectos académicos institucionales, podemos identificar distintas características del campus. En el caso estudiado —el de la UPCH—, precisamente estos rasgos se encuentran dentro de la fundamentación de los programas académicos. Estos mayormente coinciden en que la pertinencia de cada programa de formación profesional debe atender los requerimientos del mercado laboral o de proyectos.

El poder de estos organismos, en apariencia, da sentido al ámbito académico. Lo importante sería poner en el centro el desarrollo humano, para lo cual es necesaria una articulación entre los distintos niveles de gobierno, asociaciones académicas, sindicatos y redes académicas para que se cuente con la participación de decisores del gobierno, autoridades académicas, representantes sindicales, investigadores y profesores.

En este sentido, la nueva organización del campus se refleja principalmente en: a) la definición académica de la institución con un rumbo y sentido de atención al mercado de trabajo, b) el mantenimiento de la matrícula estudiantil, c) la elevación de los índice de eficiencia terminal y de titulación y d) la disminución de la deserción e índice de reprobación a costos a veces impagables. Por ejemplo, el hecho de permitir el ingreso de estudiantes sin que necesariamente demuestren contar con la formación necesaria para realizar estudios universitarios. Todo con el afán de mantener o elevar la matrícula para acceder a mayor financiamiento y ser enlistado en los rankings nacionales. Ante estas situaciones determinadas externamente a los establecimientos académicos, estos se ven en la necesidad de diseñar diversas estrategias, por ejemplo: elaboración de programas institucionales con objetivos específicos, sometimiento a procesos de evaluación y de acreditación por organismos externos y concurso en fondos extraordinarios para obtener recursos económicos adicionales. Este entorno hace que haya procesos de asimilación interna.

Los resultados del estudio ofrecen a los actores educativos una explicación amplia de las implicaciones de modelos académicos que promueven el desarrollo de competencias, desde una perspectiva sociológica. Tenemos presentes que el estudio sobre el proceso de instrumentación de los modelos innovadores en la Universidad Popular de La Chontalpa tiene un carácter exploratorio y que sería deseable, por tanto, que institucionalmente también nos 
centráramos en realizar estudios sobre las fortalezas y debilidades del proyecto académico con la finalidad de que se diseñara una propuesta de modelo universitario explícita que considerara, por lo menos, seis elementos: a) la filosofía y sociología de la educación, b) la formación axiológica, c) los ejes y enfoques pedagógicos que sustenten al modelo, d) los roles del estudiante y del profesor, e) la relación del modelo con las funciones sustantivas universitarias y f) los componentes organizativos y operativos.

\section{Referencias}

Altbach, Philip. (2014). La educación superior: un campo emergente de investigación. Diseño de políticas. En Roberta Malee y Alma Maldonado (Coords.), Organismos internacionales y políticas en educación superior: ¿pensando globalmente, actuando localmente? (pp. 23-44). México, D. F.: ANUIES-CINVESTAV-IISUE.

Bourdieu, Pierre y Wacquant, Loic. (2012). Una invitación a la Sociología reflexiva (2ª ed.). Argentina: Siglo veintiuno.

Bourdieu, Pierre. (1990). Algunas propiedades de los campos. En Sociología y cultura (pp. 135-141). México: Conaculta.

Bourdieu, Pierre. (1980). El capital social: apuntes provisionales. Barcelona, España: Anagrama.

Bourdieu, Pierre. (2000). Cosas Dichas. Barcelona, España: Gedisa.

Bourdieu, Pierre. (2001). Poder, derecho y clases sociales. Bilbao: Desclée de Brouwer.

Bourdieu, Pierre. (2002). Razones Prácticas. Barcelona, España: Anagrama.

Bourdieu, Pierre. (2003). Cuestiones de sociología. Madrid, España: Istmo.

Bourdieu, Pierre. (2013). La distinción: criterio y bases sociales del gusto. 1ra reimpresión, 1ra edición en español. México, D. F.: Taurus.

Castón, Pedro. (1996). La sociología de Pierre Bourdieu. Revista Española de Investigaciones Sociológicas, (76), 75-97.

Cobos, Verónica. (2014). Las organizaciones de la sociedad civil frente a los Objetivos de Desarrollo del Milenio: análisis de sus experiencias, limitantes y desafíos en la construcción de la agenda post-2015. En Juan Domínguez y Simone Lucatello (Coords.), Desarrollo y cooperación internacional: miradas críticas y aportes para la agenda post2015 (pp. 83-102). México, D. F.: Instituto Mora.

Delgadillo, Diana, Segura, Alejandra y Ayala, Citlali. (2012). Los actores públicos en la cooperación para el desarrollo: una aproximación conceptual y descriptiva a su ejecución en el sistema internacional. En Citlali Ayala y Jorge Pérez (coords.), Manual de cooperación internacional para el desarrollo: sus sujetos e instrumentos (pp. 107-136). México, D. F.: Instituto Mora. 
Fernández, José Manuel y Puente, Aníbal. (2009). La noción de campo en Kurt Lewin y Pierre Bourdieu: un análisis comparativo. Revista Española de Investigaciones Sociológicas, (127), 33-53.

García, José Luís, González, María Ángeles y Ballesteros, Belén. (2001). Introducción a la investigación en Educación (Tomo I). Madrid, España: UNED.

Giddens, Anthony y Sutton, Philip. (2015). Conceptos esenciales de Sociología. Madrid, España: Alianza editorial.

Giddens, Anthony. (2010). Sociología (6ta. ed.). Madrid, España: Alianza editorial.

Ibarra, Eduardo. (2001). La universidad en México hoy: gubernamentalidad y modernización. México, D. F.: UNAM.

Miranda, Fernando. (2001). Las universidades como organizaciones del conocimiento: el caso de la Universidad pedagógica Nacional. México, D. F.: COLMEX-UPN.

Muñoz, Humberto (coord.). (2002). Universidad: política y cambio institucional. México, D. F.: CESU-UNAM.

Powell, Walter y Dimaggio, Paul. (2001). Retorno a la jaula de hierro: el isomorfismo institucional y la racionalidad colectiva en los campos organizacionales. En Walter Powell y Paul Dimaggio (Comps.), El nuevo institucionalismo en el análisis organizacional (pp. 104-125). México, D. F.: CNCP-UAEM-FCE.

Sánchez-Dromundo, Rosalba Angélica. (2007). La teoría de los campos de Bourdieu, como esquema teórico de análisis del proceso de graduación en posgrado. Revista Electrónica de Investigación Educativa, 9(1), 1-21. Recuperado de http://redie.uabc.mx/redie/article/view/161

Universidad Autónoma de Nuevo León. (2012). Plan de Desarrollo Institucional. Universidad Autónoma de Nuevo León 2012-2020 Versión 1.0.UANL. México: UANL. Recuperado de https://goo.gl//OHhkm

Universidad Autónoma de Queretano. (2007). Plan Institucional de Desarrollo 2007-2012. Universidad Autónoma de Querétaro. México: UAQ. Recuperado de http://www.uaq.mx/planeacion/pide/pide2007-2012.pdf

Universidad de Guanajuato. (2011). Modelo Educativo de la Universidad de Guanajuato. Universidad de Guanajuato. México: UG. Recuperado de https://goo.gl/Cd2xxh

Universidad Juárez Autónoma de Tabasco. (2012). Plan de Desarrollo Institucional 2012-2016. México: UJAT. Recuperado de https://goo.gl/nXDNCW

Unison. (2014). Plan de Desarrollo Institucional 2013-2017. Un compromiso con el desarrollo sustentable, la calidad y la pertinencia social. Universidad de Sonora. México. Unison. Recuperado de https://goo.gl/ygy3Q6

Velasco, David. (1998). La fórmula generadora del sentido práctico. Una aproximación a la filosofía de la práctica de Pierre Bourdieu. Revista Espiral, 4(12), 33-80. 